\title{
Lipid-laden macrophages in induced sputum are a marker of oropharyngeal reflux and possible gastric aspiration
}

\author{
K. Parameswaran*, M. Anvari**, A. Efthimiadis*, D. Kamada*, F.E. Hargreave*, C.J. Allen*
}

\begin{abstract}
Lipid-laden macrophages in induced sputum are a marker of oropharyngeal reflux and possible gastric aspiration. K. Parameswaran, M. Anvari, A. Efthimiadis, D. Kamada, F.E. Hargreave, C.J. Allen. (C)ERS Journals Ltd 2000.

ABSTRACT: The diagnostic properties of a "lipid index" of macrophages in induced sputum as a noninvasive marker of aspiration of acidic gastric contents were evaluated.

In a cross-sectional study, 33 subjects (17 with symptoms suggestive of gastrooesophageal reflux) with normal chest radiographs and no symptoms of aspiration or sinus disease, underwent dual-channel 24-h ambulatory oesophageal $\mathrm{pH}$ recording and sputum induction. Oropharyngeal reflux, defined as at least one episode of a fall in $\mathrm{pH}$ to $<4$ at the upper oesophageal electrode, was considered indicative of aspiration of acidic gastric contents ("gold standard"). An index for the presence of intracellular lipid in sputum macrophages, detected by oil red $O$ stain, was obtained. The sensitivity, specificity and predictive values of this "lipid index" were calculated.

The "lipid index" could be calculated in 29 of 33 samples with high interobserver repeatability (intraclass correlation coefficient 0.96 ). Twenty subjects showed oropharyngeal reflux and nine did not. The median "lipid index" in subjects with oropharyngeal reflux (24.5) was significantly greater than that in those without reflux (1.0) (p<0.001). A "lipid index" of 7.0 had a sensitivity of $90 \%$, a specificity of $89 \%$, a positive predictive value of $95 \%$ and a negative predictive value of $80 \%$.

A "lipid index" of 7.0 in the macrophages of induced sputum is a good marker of oropharyngeal reflux.

Eur Respir J 2000; 16: 1119-1122.
\end{abstract}

Reflux of acidic gastric contents causing parenchymal and interstitial lung disease is thought to be common $[1,2]$, particularly in elderly debilitated patients [3] and children $[4,5]$. Gastro-oesophageal reflux (GOR) may be associated with pulmonary microaspiration [6] and may also contribute to the pathogenesis of cough $[7,8]$ and asthma [9]. There is presently no noninvasive test for the detection of aspiration of gastric juice into the airway or the assessment of the success of antireflux treatment. Demonstration of lipid in alveolar macrophages may be an indicator of aspiration of acidic gastric contents [10], and cytological examination of bronchoalveolar lavage fluid for lipid-laden macrophages has been suggested as a useful diagnostic test for aspiration [11-15]. Determination of cell counts in induced sputum separated from saliva is a reliable noninvasive method of assessing airway inflammation [16], and the use of an index of macrophages in sputum staining positive for intracellular lipid has been reported [17]. In previous studies there have been two difficulties, the lack of a "gold standard" for the diagnosis of aspiration and the inclusion of patients with a variety of chronic respiratory diseases [18]. Therefore, the present study investigated the diagnostic value of an index of sputum lipid-laden macrophages in identifying acid aspiration, using dual-channel 24-h $\mathrm{pH}$ monitoring, in subjects without any chronic respiratory disorder other than asthma.
Depts of *Medicine and **Surgery, St Joseph's Hospital and McMaster University, Hamilton, Ontario, Canada.

Correspondence: C.J. Allen

Firestone Regional Chest \& Allergy Clinic St Joseph's Hospital

50 Charlton Avenue East

Hamilton

Ontario L8N 4A6

Canada

Fax: 19055216158

Keywords: Acid aspiration gastro-oesophageal reflux induced sputum

lipid-laden macrophages

Received: February 182000

Accepted after revision July 282000
Subjects and methods

\section{Subjects}

Thirty-three adult subjects who consented to undergo dual-channel 24-h ambulatory $\mathrm{pH}$ recording were recruited from the patients and staff of the Firestone Chest and Allergy and the Gastro-intestinal Motility Disorders Clinics of St Joseph's Hospital (table 1). Seventeen subjects had symptoms suggestive of GOR and 16 did not. None had a history suggestive of aspiration, had a lipid storage disease or fat embolism, or were receiving nutritional supplementation with fat emulsions. All had normal chest radiographs. No patient had experienced any occupational dust exposure or known exposure to moulds. None were taking nasal medications. Seventeen subjects had mild asthma (10 atopics, mean \pm SD forced expiratory volume in one second (FEV1) $82.9 \pm 13.8 \%$ of the predicted value, geometric mean \pm SD provocative concentration of methacholine causing a $20 \%$ fall in FEV1 $1.7 \pm 2.8$ $\mathrm{mg} \cdot \mathrm{mL}^{-1}$. Five of them were on inhaled corticosteroids (median dose $500 \mu \mathrm{g}$ equivalent of beclomethasone, range $50-800 \mu \mathrm{g})$. None of the subjects had any other respiratory problem. The study was approved by the Ethics Committee of St Joseph's Hospital and all subjects gave written informed consent. 
Table 1. - Subject characteristics, oesophageal $\mathrm{pH}$ and induced sputum indices

\begin{tabular}{lcc}
\hline & Oropharyngeal reflux present & Oropharyngeal reflux absent \\
\hline Subjects n & 20 & 9 \\
Males n & 9 & 2 \\
Age yrs & $40 \pm 16$ & $34 \pm 12$ \\
GOR symptoms n* & 10 & 3 \\
Confirmed GOR diagnosis n & 14 & 2 \\
Asthma n & 9 & 8 \\
FEV1 \% pred & $90.2 \pm 15.9$ & $94.4 \pm 9.5$ \\
Lower oesophagus pH monitor & & $2.9 \pm 3.1$ \\
Cumulative time at pH <4\% & $5.9 \pm 6.2$ & $74.1 \pm 80.4$ \\
Reflux episodes n & $84.5 \pm 95.6$ & 0 \\
Upper oesophagus pH monitor & & 0 \\
Cumulative time at pH $<4 \%$ & $0.5 \pm 0.9$ & $4.7 \pm 10.3$ \\
Reflux episodes n & $14.8 \pm 12.5$ & $1.0(0-28)$ \\
Sputum lipid index & $35.1 \pm 29.1$ & $5.7 \pm 5.8$ \\
& $24.5(4-100)$ & $38.6 \pm 23.9$ \\
Sputum cell count & & $1.7 \pm 2.9$ \\
Total 10 cells: ${ }^{-1}$ & $3.0 \pm 1.6$ & $57.0 \pm 22.0$ \\
Neutrophils \% & $35.8 \pm 20.2$ & $1.2 \pm 0.8$ \\
Eosinophils \% & $1.5 \pm 2.9$ & \\
Macrophages \% & &
\end{tabular}

Data are presented as mean \pm SD or median (range). *: four excluded patients showed symptoms of gastro-oesophageal reflux (GOR); ${ }^{\#}$ : total number not the number staining positive for lipids. FEV1: forced expiratory volume in one second; \% pred: percentage of the predicted value.

\section{Methods}

The present investigation was a cross-sectional singlecentre, observational study. All subjects underwent oesophageal motility and $\mathrm{pH}$ studies and sputum examination and completed a GOR symptom questionnaire within one week of each other. Symptoms of GOR were assessed using a validated questionnaire [19]. Oesophageal measurements were made after stopping any antireflux medications for $\geq 5$ days. An ambulatory dual-channel digitrapper (Medtronic-Synectics, Stockholm, Sweden) was used to perform the 24-h $\mathrm{pH}$ testing. The lower $\mathrm{pH}$ electrode was positioned $5 \mathrm{~cm}$ above, and the upper $\mathrm{pH}$ electrode $25 \mathrm{~cm}$ above, the manometrically determined lower oesophageal sphincter. The following measurements were made using both electrodes: cumulative time (expressed as a percentage of the total recording time) during which the $\mathrm{pH}$ was $<4.0$; and the number of episodes during which the $\mathrm{pH}$ fell $<4.0$. GOR was defined as a cumulative time of $>4 \%$ of the recording time with a $\mathrm{pH}$ of $<4.0$ at the lower $\mathrm{pH}$ electrode. Oropharyngeal reflux was defined as at least one episode of a fall in $\mathrm{pH}$ to $<4.0$ at the upper $\mathrm{pH}$ electrode, not associated with eating or drinking. This was considered to be an indicator of possible aspiration of acidic gastric contents.

Sputum was induced with hypertonic saline, and then separated from saliva and processed within $2 \mathrm{~h}$; cell counts were determined as previously described [16]. Cytospins were prepared and stained with oil red O (Sigma-Aldrich Canada Ltd, Oakville, Ontario, Canada) to detect cytoplasmic lipid droplets. One hundred consecutive macrophages were evaluated using a five-point scale similar to that reported by CoRwIN and IRWIN [11] (0: absence of staining; 1: one or a few lipid droplets; 2: many distinct droplets; 3: many droplets with visible nucleus; and 4: many droplets completely covering the cytoplasm and obscuring the nucleus). The "lipid index" was the sum of the scores for 100 consecutive macrophages and thus had a possible range of 0-400. Assessments were carried out by two independent observers (high interobserver agreement with an intraclass correlation coefficient of 0.96), who were blind to the clinical details of the subjects.

\section{Analysis}

All data were analysed using the statistical package SPSS for Windows $\AA$, release 10.0 (Statistical Package for the Social Sciences, Inc., Chicago, IL, USA). The sensitivity, specificity and predictive values of the lipid index in the detection oropharyngeal reflux were calculated from the receiver operating characteristic curve, constructed for different cut-off values of the index. Betweengroup comparisons were made using the Mann-Whitney U-test. Correlations between variables were examined using Spearman's coefficient of rank correlation.

\section{Results}

The lipid index could not be calculated in four of the 33 sputum samples because of poor quality of staining; these four subjects were excluded from subsequent analysis. The demographic, $\mathrm{pH}$ and sputum data (table 1) of the 29 included subjects were evaluated. There were 20 subjects with at least one episode of reflux to the oropharynx. The lipid index in these subjects was significantly greater $(p<0.001)$ than that in those without reflux, and there was a significant correlation between the lipid-index and the number of reflux episodes $(\mathrm{rS}=0.5, \mathrm{p}<0.01)$. The receiver operating characteristic curve (fig. 1) showed a lipid index of 7.0 to the maximum area under the curve (upper left corner), with a sensitivity of $90 \%$, a specificity of 


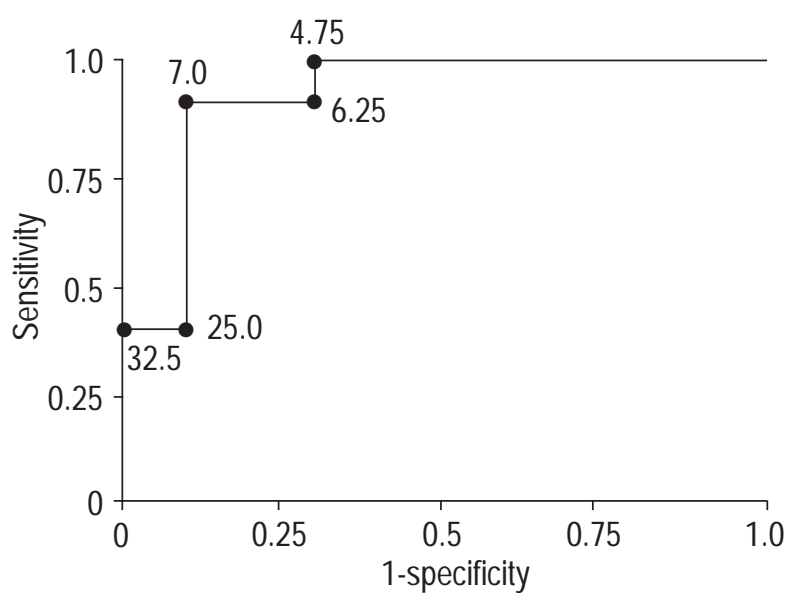

Fig. 1. - Receiver operating characteristic curve for the lipid index of macrophages in induced sputum for the detection of oropharyngeal reflux measured by dual-channel 24-h ambulatory oesophageal $\mathrm{pH}$ recording. Numbers corresponding to dots represent lipid index of macrophages.

$89 \%$, a positive predictive value of $95 \%$ and a negative predictive value of $80 \%$ (table 2 ). The only difference between the asthmatic and nonasthmatic subjects was in sputum eosinophil count (mean \pm SD 3.6-4.0 and 0.2 \pm $1.2 \%)$ respectively, $\mathrm{p}<0.001)$. There were no significant differences in lipid index (median interquartile range) 16 (33) in asthmatics and 15.5 (25) in nonasthmatics. The total and neutrophil differential cell counts in sputum, the 24-h pH scores and the number of reflux episodes in both the lower and upper oesophagus were also similar in asthmatic and nonasthmatic subjects.

\section{Discussion}

In the present pilot study, it has been shown that a lipidladen macrophage index of 7.0 in induced sputum from subjects with a normal chest radiograph and no clinical history to suggest pulmonary aspiration is a sensitive $(90 \%)$ and specific $(89 \%)$ indicator of oropharyngeal reflux, with high positive $(95 \%)$ and negative $(80 \%)$ predictive values.

The presence of lipid in alveolar macrophages is a consequence of aspiration of gastric contents or exogenous causes of lipoid pneumonia [20]. In the present study, the latter possibility was excluded by including only subjects with normal chest radiographs. The staining method and semiquantitiative index used are the same as previously reported by CoRwIN and IRWIN [11]. From the available literature, it is difficult to determine the measurement properties of this index because there is uncertainty regarding the gold standard for aspiration [18]. Most studies have used relatively crude indicators such as response to therapy, clinically witnessed aspiration, barium oesophagography or conventional single-channel 24-h pH monitoring to detect aspiration [11-13, 15, 21]. Some studies have used symptoms alone [17], which are insensitive in the diagnosis of oropharyngeal reflux and GOR. In the present study, dual-channel oesophageal $\mathrm{pH}$ recording was used to detect oropharyngeal reflux.

A number of previous studies have attempted to detect aspiration by examining sputum $[15,17]$, tracheobronchial
Table 2. - Evaluation of the sputum lipid index diagnostic test $^{*}$

\begin{tabular}{lccc}
\hline & $\begin{array}{c}\text { Oropharyngeal } \\
\text { reflux present }\end{array}$ & $\begin{array}{c}\text { Oropharyngeal } \\
\text { reflux absent }\end{array}$ & Total \\
\hline Lipid index $>7$ & 18 & 1 & 19 \\
Lipid index $<7$ & 2 & 8 & 10 \\
Total & 20 & 9 & 29 \\
\hline
\end{tabular}

*: $\mathrm{n}=29$. Prevalence (pre-test likelihood): 69\%; Sensitivity: 90\%; Specificity: 89\%; Positive predictive value (post-test likelihood of oropharyngeal reflux): $95 \%$; Negative predictive value (post-test likelihood of oropharyngeal reflux despite negative test): $80 \%$; Likelihood ratio of a positive test $(95 \%$ confidence interval CI): 8.1 (2.0-45.4); Likelihood ratio of a negative test $(95 \% \mathrm{CI}): 0.1(0.03-0.36)$.

washings [14] or bronchoalveolar lavage fluid [11-13, 21] for the presence of lipid in macrophages. In those studies that included healthy controls [22], lipid-laden macrophages were seldom found and therefore the lipid index was low (mean \pm SD 1.31.5). A lipid index of 85-100 in bronchoalveolar lavage fluid has been suggested to be a sensitive indicator of parenchymal disease associated with clinically evident macroaspiration $[11,21]$. The significance of lower values is less clear. The lower lipid indexes observed with induced sputum, as compared to previous studies using bronchoalveolar lavage, may be related to a number of factors. First, none of the present subjects had a clinical history of aspiration and all had normal chest radiographs; therefore micro- rather than macroaspiration was being identified. Secondly, the previous studies sampled focal areas of overt disease characterized by an abnormality on the chest radiograph where it would be expected that the lipid index would be high. In contrast, sputum induction, which samples both lungs diffusely, was used. The authors believe that the present results from induced sputum are valid because the index correlated with the number of episodes of reflux to the oropharynx. The index may be useful clinically for the identification of oropharyngeal reflux, and presumably microaspiration, associated with gastro-oesophageal reflux in patients with respiratory symptoms.

Acknowledgements. The authors would like to thank S. Weston for the sputum cell counts and the staff of the Gastro-intestinal Motility Disorders Laboratory (St. Joseph's Hospital, Hamilton) for the oesophageal measurements and $\mathrm{N}$. Jones (McMaster University, Hamilton) for reviewing the manuscript.

\section{References}

1. Allen CJ, Newhouse MT. Gastroesophageal reflux and chronic respiratory disease. Am Rev Respir Dis 1984; 129 : 645-647.

2. Tobin RW, Pope CE 2nd, Pellegrini CA, Emond MJ, Sillery J, Raghu G. Increased prevalence of gastroesophageal reflux in patients with idiopathic pulmonary fibrosis. Am J Respir Crit Care Med 1998; 158: 18041808. 
3. Raiha I, Manner R, Hietanen E, Hartiala J, Sourander L. Radiographic pulmonary changes of gastro-esophageal reflux in elderly patients. Age Ageing 1992; 21: 250-255.

4. Buts JP, Barudi C, Moulin D, Claus D, Cornu G, Otte B. Prevalence and treatment of silent gastro-esophageal reflux in children with recurrent respiratory disorders. Eur J Pediatr 1986; 145: 396-400.

5. Malfroot A, Vandenplas Y, Verlinden M, Piepsz A, Dab I. Gastro-esophageal reflux and unexplained chronic respiratory disease in infants and children. Pediatr Pulmonol 1987; 3: 208-213.

6. Pelligrini CA, DeMeester TR, Johnson LF, Skinner DB. Gastroesophageal reflux and pulmonary aspiration: incidence, functional abnormality, and results of surgical therapy. Surgery 1979; 86: 110-119.

7. Irwin RS, Zawacki JK, Curley FJ, French CL, Hoffman PJ. Chronic cough as the sole presenting manifestation of gastroesophageal reflux. Am Rev Respir Dis 1989; 140: 1294-1300.

8. Allen CJ, Anvari M. Gastro-esophageal reflux related cough and its response to laparoscopic fundoplication. Thorax 1998; 53: 963-968.

9. Harding SM. Gastroesophageal reflux and asthma: insight into the association. J Allergy Clin Immunol 1999; 104: 251-259.

10. Sundberg RH, Kirschner KE, Brown MJ. Evaluation of lipoid pneumonia. Dis Chest 1959; 36: 594-601.

11. Corwin RW, Irwin RS. The lipid-laden alveolar macrophage as a marker of aspiration in parenchymal lung disease. Am Rev Respir Dis 1985; 132: 576-581.

12. Ahrens P, Noll C, Kitz R, Willigens P, Zielen S, Hofmann D. Lipid-laden macrophages (LLAMs): a useful marker of silent aspiration in children. Pediatr Pulmonol 1999; 28: $83-88$

13. Nussbaum E, Maggi JC, Mathis R, Galant SP. Associ- ation of lipid-laden alveolar macrophages and gastroesophageal reflux in children. J Pediatr 1987; 110: 190-194.

14. Reclade AL, Nickerson BG, Vegas M, Scott CB, Landing $\mathrm{BH}$, Warburton D. Lipid-laden macrophages in tracheal aspirates of newborn infants receiving intravenous lipid infusions: a cytologic study. Pediatr Pathol 1984: 2: 2534.

15. Vejar L, Le Cerf P. Pulmonary aspiration in children. Quantification of lipid laden alveolar macrophages. Rev Med Chil 1997; 125: 191-194.

16. Pizzichini E, Pizzichini MM, Efthimiadis A, et al. Indices of airway inflammation in induced sputum: reproducibility and validity of cell and fluid-phase measurements. Am J Respir Crit Care Med 1996; 154: 308-317.

17. Carney IK, Gibson PG, Murree-Allen K, Saltos N, Olson LG, Hensley MJ. A systematic evaluation of mechanisms in chronic cough. Am J Respir Crit Care Med 1997; 156: 211-216.

18. Colombo JL, Hallberg TK. Pulmonary aspiration and lipid-laden macrophages: in search of gold (standards). Pediatr Pulmonol 1999; 28: 79-82.

19. Parameswaran K, Allen CJ, Belda J, Anvari M. Reproducibility, validity and responsiveness of a GERD specific symptom score. Gastroenterology 1999; 116: A45-A46.

20. Wright JL. Consequences of aspiration and bronchial aspiration. In: Thurlbeck WM, Churg AM, eds. Pathology of the Lung. New York, Thieme Medical Publishers, Inc, 1995; pp. 1111-1127.

21. Bauer ML, Lyrene RK. Chronic aspiration in children: evaluation of the lipid-laden macrophage index. Pediatr Pulmonol 1999; 28: 94-100.

22. Schellhase DE, Fawcett DD, Schutze GE, Lensing SY, Tryka AF. Clinical utility of flexible bronchoscopy and bronchoalveolar lavage in young children with recurrent wheezing. J Pediatr 1998; 132: 312-318. 